\title{
NOTA SOBRE LA DISTRIBUCIÓN GEOGRÁFICA Y MORFOLOGÍA DE MANFREDA INVOLUTA (AGAVACEAE)
}

\author{
Aarón Rodríguez ${ }^{1}$, Pablo Carrillo-Reyes ${ }^{2}$ y Arturo Castro-Castro ${ }^{1}$ \\ ${ }^{1}$ Universidad de Guadalajara, Instituto de Botánica, Departamento de Botánica y \\ Zoología, Centro Universitario de Ciencias Biológicas y Agropecuarias \\ Apdo. postal 1-139, 45101 Zapopan, Jalisco, México. rca08742@cucba.udg.mx \\ ${ }^{2}$ Instituto de Ecología, A.C., Departamento de Biología Evolutiva, km. 2.5 carretera \\ antigua a Coatepec, 91070 Xalapa, Veracruz, México.
}

\section{RESUMEN}

Como resultado de recientes colectas botánicas en el occidente de México, se amplía la distribución geográfica conocida y se complementa la descripción morfológica de Manfreda involuta, una especie endémica del occidente de México.

Palabras clave: Agavaceae, Manfreda, oeste de México.

\section{ABSTRACT}

As a result of recent botanical collections in western Mexico, the known geographical distribution of Manfreda involuta is extended and its morphological description completed. The species is endemic to western Mexico.

Key words: Agavaceae, Manfreda, western Mexico.

\section{INTRODUCCIÓN}

La familia Agavaceae Endl. tiene una distribución geográfica restringida al continente americano. En México, se reconocen al menos 342 especies agrupadas en ocho géneros: Agave L., Beschorneria Kunth, Furcraea Vent., Hesperaloe Engelm., Manfreda Salisb., Polianthes L., Prochnyanthes S. Watson y Yucca L. (GarcíaMendoza y Galván, 1995). Recientemente, algunos autores incluyen a Hesperoyucca (Engelm.) Baker en la misma familia (Good-Avila et al., 2006; Bogler et al., 2006). 
Manfreda se distribuye desde el este y sureste de los Estados Unidos de América (Virginia, Maryland, Indiana, Missouri, Arkansas, Florida y Texas) hasta Honduras y El Salvador (Rocha et al., 2006; Verhoek-Williams, 1978; Williams, 1998). El grupo está formado por más de 27 especies (Espejo y López-Ferrari, 1993; GarcíaMendoza y Galván, 1995; Piña-Luján, 1985) y al igual que el resto de los géneros de agaváceas, México tiene la mayor diversidad de especies con 26 de ellas (GarcíaMendoza y Galván, 1995; Williams, 1998).

Las relaciones filogenéticas entre Agave, Manfreda, Polianthes y Prochnyanthes no han sido determinadas (Bogler et al., 1995, 2006; Bogler y Simpson, 1995, 1996; Clary y Simpson, 1995; Good-Avila et al., 2006; Hernández-Sandoval, 1995). En todos los análisis, el género Agave aparece como un grupo parafilético y para resolver el problema Thiede y Eggli (1999) propusieron un concepto más amplio de Agave en el que incluyeron a Manfreda, Polianthes y Prochnyanthes. En consecuencia, M. involuta podría ser considerada como Agave involuta (McVaugh) Thiede \& Eggli. Sin embargo, creemos que la propuesta es precipitada y por el momento optamos por apegarnos al criterio de Williams (1998).

Manfreda involuta McVaugh es una especie poco conocida y con una distribución geográfica restringida a la porción sur de la Sierra Madre Occidental y el extremo occidental del Eje Volcánico Transversal. Fue descrita por McVaugh en 1989 con base en dos colecciones. El tipo se recolectó en el municipio de Mezquitic, Jalisco y el único parátipo se citó como "Sierra du Nayarit (Territoire Huichol), Etat de Jalisco" (McVaugh 1989). Los frutos y las semillas no fueron descritos en el protólogo por falta de material. Colectas recientes documentan la presencia de M. involuta en los estados de Zacatecas, Nayarit y Jalisco. El material nuevo tiene flores, frutos y semillas que fueron utilizados para complementar su descripción morfológica.

\section{RESULTADOS Y DISCUSIÓN}

Manfreda involuta McVaugh, Flora Novo-Galiciana 15: 231-232. 1989. Tipo: México, Jalisco, municipio de Mezquitic, near San Andrés Cohamiata, Bauml \& Voss 1466, (holótipo en RSA, isótipo en MICH). (Figs. 1 y 2).

Hierba perenne de 0.6-1.8 m de alto; planta con rizoma erecto, cónico, $2.5 \mathrm{~cm}$ de alto, 1-2 cm de diámetro en la base; restos basales de las hojas membranáceos, 5-8 $\mathrm{cm}$ de longitud; raíces carnosas y fibrosas; hojas (con frecuencia ausentes durante la floración) 6-11, lineares, $18-80 \mathrm{~cm}$ de largo, 2-9 mm ancho, plegadas o involutas en su punto medio entonces pareciendo tubulares, en cuyo caso miden 2-3 mm de diámetro, de color verde claro, sin máculas, papilosas sobre los márgenes hialinos y sobre las nervaduras del envés; inflorescencia de 0.6-1.8 $\mathrm{m}$ de alto, erecta a lige- 
ramente arqueada; pedúnculo de 0.5-1.3 m de largo, brácteas del pedúnculo 6-9, escariosas, ovado-acuminadas o caudadas, 1-7.5 cm largo, 0.4-1 cm de ancho; porción floral de 10-35 cm de largo, laxa, 3-18 flores; brácteas florales primarias más cortas que el ovario, escariosas, lanceolado-ovadas, atenuadas, 4-10 mm de largo, 2-4 mm de ancho; bractéolas de forma igual que las brácteas primarias pero más pequeñas, 2-3 mm de largo, glaucas; flores sésiles, ascendentes cuando maduras; perianto verdoso con tintes purpúreos, tubo infundibuliforme, más largo que los lóbulos, 1.2-1.5 $\mathrm{cm}$ de largo, 5-7 mm de diámetro; lóbulos oblongos, erectos, 7-8 mm de largo, 3-5 $\mathrm{mm}$ de ancho; filamentos de color púrpura, $1.2-1.8 \mathrm{~cm}$ de largo, excediendo la longitud del tubo por 3-9 $\mathrm{mm}$; anteras de 7-8.5 $\mathrm{mm}$ de largo y de $1-1.2 \mathrm{~mm}$ de ancho; estilo 2.8-3.3 cm de largo, excediendo la longitud del tubo por $1-1.5 \mathrm{~cm}$, de color púrpura oscuro; estigma trilobado del mismo color que el estilo; ovario elipsoidal, $10 \mathrm{~mm}$ de largo, $2.5 \mathrm{~mm}$ de diámetro; cápsula globosa o subglobosa, subestipitada (en A. Rodríguez y A. L. Pérez-Álvarez 4852, IBUG), 1.3-1.5 cm de largo, 1.1-1.3 $\mathrm{cm}$ de diámetro, algunas veces proyectada en el ápice en un cono de $1.5-2.5 \mathrm{~mm}$ de largo,1.0-1.5 mm de diámetro, glauco, perianto persistente, marca del perianto remanente formando un anillo, 3.2-3.7 $\mathrm{mm}$ de diámetro; semillas deltoides, negras, brillantes, 4.8-6.0 $\mathrm{mm}$ de largo, 4-5 $\mathrm{mm}$ de ancho.

Distribución y hábitat: Manfreda involuta crece en el sudeste de Nayarit, norte y oeste de Jalisco y el sur de Zacatecas (Fig. 3). Habita en paredones o laderas rocosas de poca pendiente en encinares y bosques de pino y encino, a una altitud de 740 a $2000 \mathrm{~m}$.

Fenología: La planta florece de marzo a julio y fructifica de abril a julio. Al igual que todas las especies de Manfreda, M. involuta es una especie protándrica. En la etapa de apertura de las anteras, el estilo es más corto que los estambres, pero después cuando llega a su maduración crece hasta sobrepasarlos. En M. involuta es común encontrar individuos con flores pero sin hojas o con éstas muy pequeñas $\mathrm{y}$ filiformes (A. Rodríguez y C. Briseño 3660, A. Rodríguez 3107). Más tarde durante la época de lluvias, en el verano, las hojas se desarrollan hasta alcanzar la forma y el tamaño descritos.

Especímenes examinados: Jalisco: municipio de Cabo Corrientes, km 175 carretera Méx. 200 entre El Tuito y Puerto Vallarta, $36.5 \mathrm{~km}$ al S de Puerto Vallarta, justo en la intersección con la brecha que conduce a El Cuale, alt. $743 \mathrm{~m}, 20^{\circ} 21^{\prime} \mathrm{N}$, $105^{\circ} 19^{\prime}$ W, 24.I.2006 estéril, A. Rodríguez et al. 4823 (CHAPA, ENCB, IBUG, IEB, MEXU); ibid., 26.IV.2006 fl. fr., A. Rodríguez y A. L. Pérez-Álvarez 4852 (ENCB, IBUG, IEB, MEXU). Nayarit: municipio de Santa María del Oro, km 190 carretera libre Méx. 15 entre Guadalajara y Tepic, alt. 1279 m, 21 ${ }^{\circ} 18.03^{\prime} \mathrm{N}, 104^{\circ} 39.08^{\prime} \mathrm{W}$, 


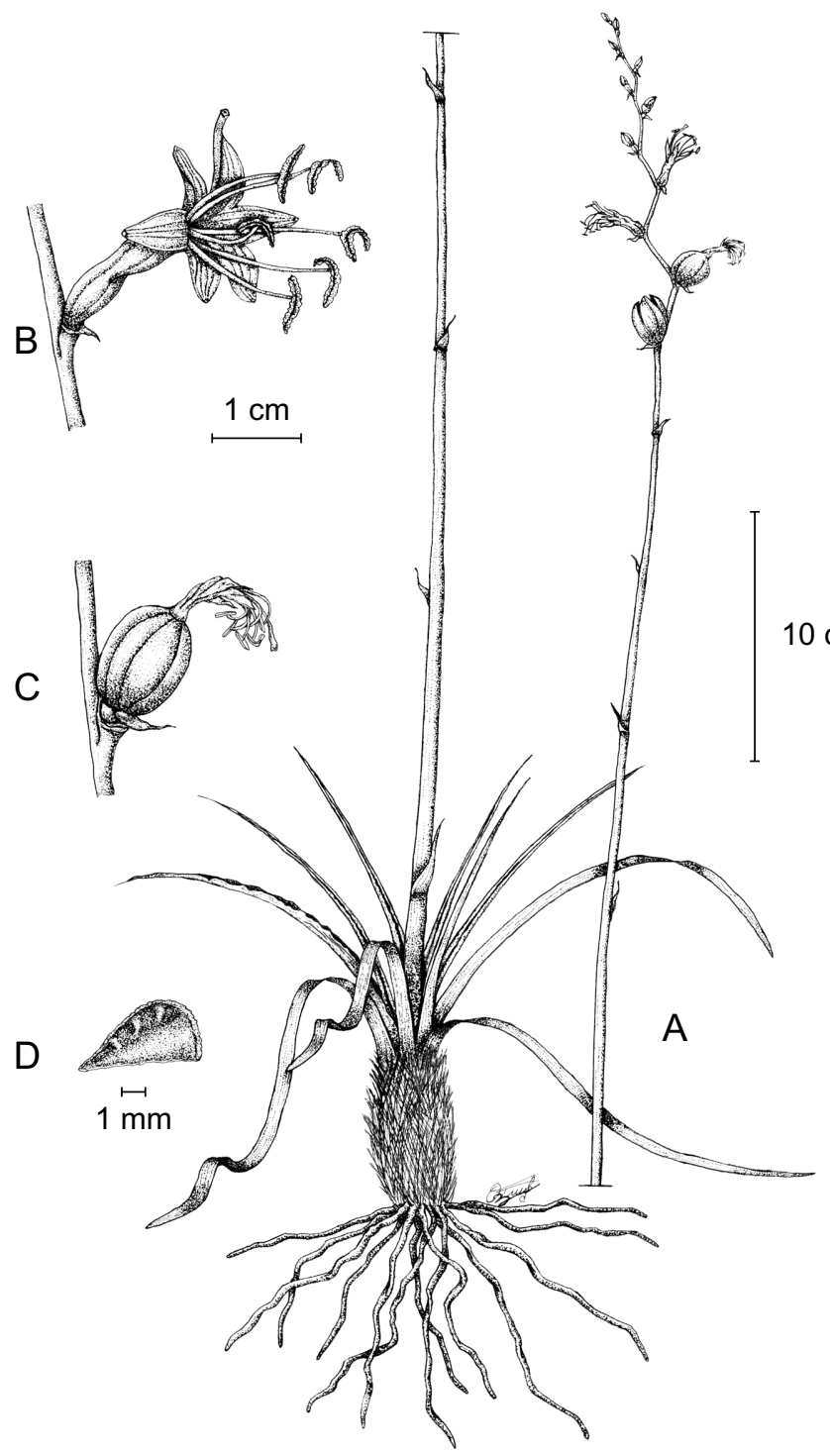

Fig. 1. Manfreda involuta. A. hábito; B. flor; C. fruto; D. semilla. (A. Rodríguez \& A. L. Pérez-Álvarez 4852). 

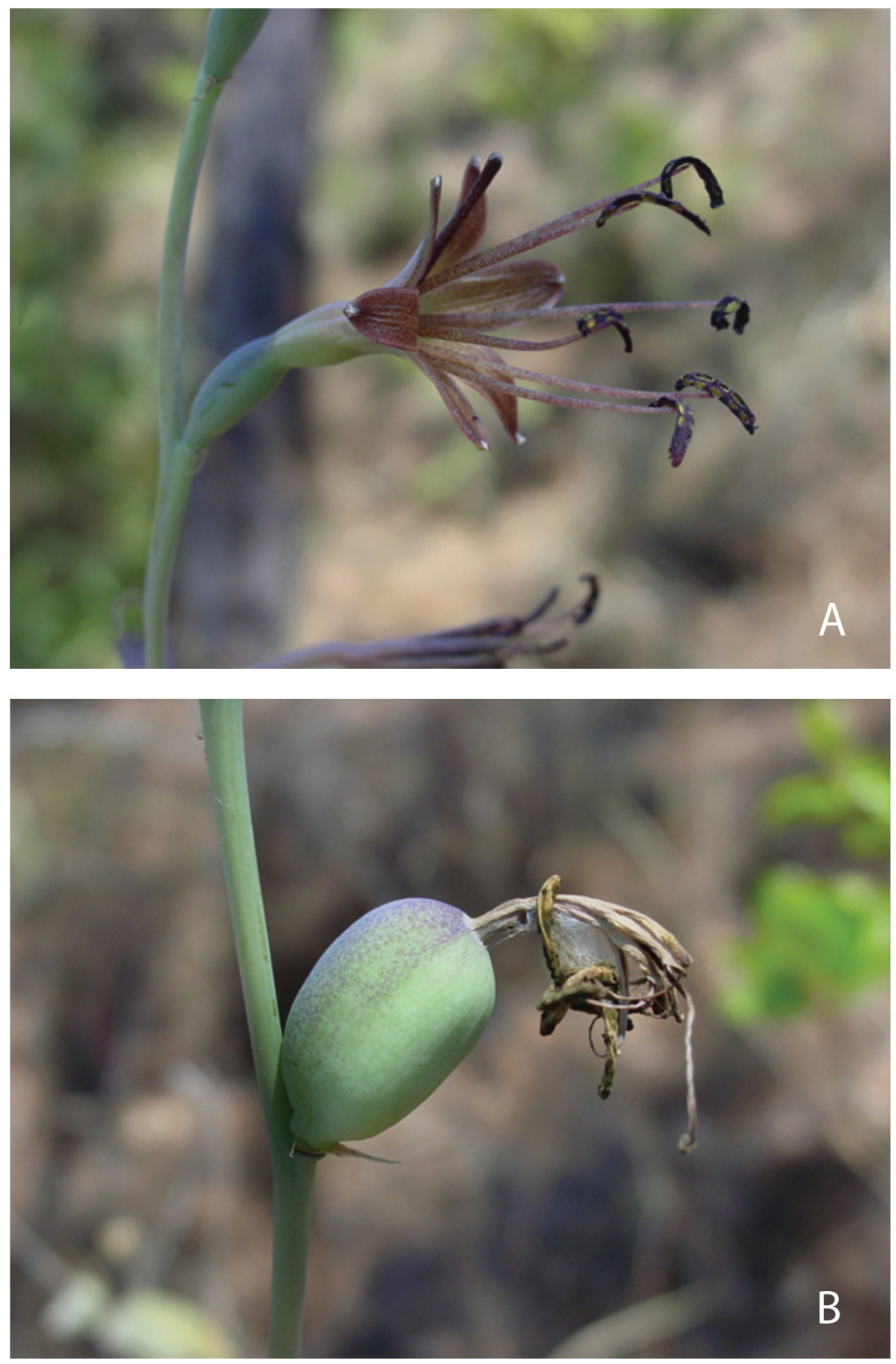

Fig. 2. Manfreda involuta. A. flor. B. fruto (A. Rodríguez y A. L. Pérez-Álvarez 4852, IBUG).

13.VII.2003 fl., A. Rodríguez 3107 (CHAPA, ENCB, IBUG, IEB, MEXU, XAL); ibid., 5.VII.2004 fl., A. Rodríguez y C. Briseño 3660 (IBUG, IEB, MEXU, XAL). Zacatecas: municipio de Teúl de González Ortega, $0.5 \mathrm{~km}$ al S de Huitzila por el camino a La Lobera, alt. $1720 \mathrm{~m}, 21^{\circ} 12^{\prime} \mathrm{N}, 103^{\circ} 36^{\prime} \mathrm{W}$, 9.IV.2001. fl., P. Carrillo-Reyes y E. M. Barba 1631 (IBUG, MEXU). 
Manfreda involuta es única en su carácter de producir hojas filiformes después del período de fructificación. El tiempo en que florece se traslapa con el de $M$. jalisca$n a$ que ocurre de octubre a abril. Poblaciones de ambas especies han sido colectadas cerca una de otra en el sur de Nayarit y en el norte de Jalisco (McVaugh, 1989).

\section{AGRADECIMIENTOS}

Los autores agradecen a Osvaldo Zuno la elaboración de la figura 1. También deseamos expresar nuestra gratitud a E. M. Barba Robert, Luis Ortiz Catedral y Christian Briseño Avena por su ayuda durante el trabajo de campo.

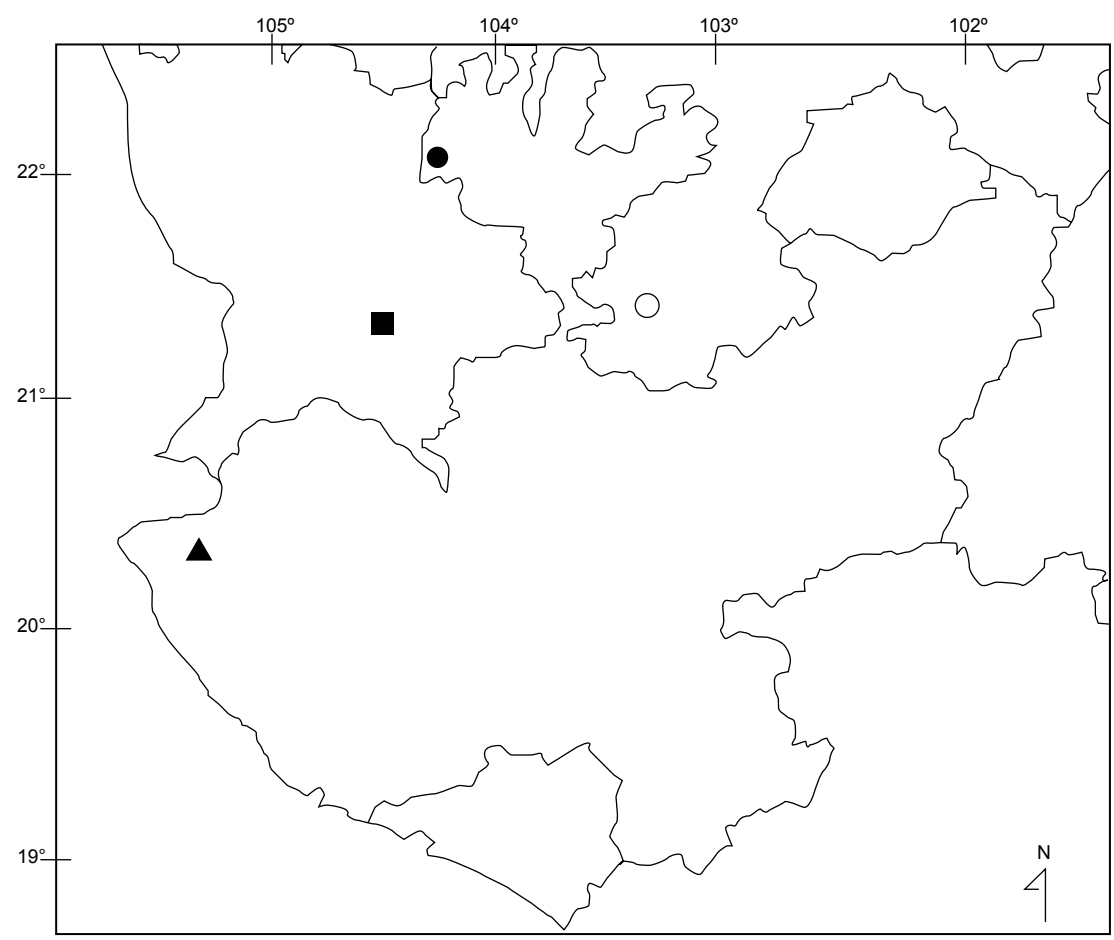

Fig. 3. Distribución geográfica conocida de Manfreda involuta. ( $\mathbf{\Lambda}$ ) Cabo Corrientes, Jalisco; (•) Santa María del Oro, Nayarit; (०) Teúl de González Ortega, Zacatecas; (•) localidad tipo. 


\section{LITERATURA CITADA}

Bogler, D. J. y B. B. Simpson. 1995. A chloroplast DNA study of the Agavaceae. Syst. Bot. 20: 191-205.

Bogler, D. J. y B. B. Simpson. 1996. Phylogeny of Agavaceae based on its rDNA sequence variation. Amer. J. Bot. 83: 1225-1235.

Bogler, D. J., J. L. Neff y B. B. Simpson. 1995. Multiple origins of the yucca-yucca moth association. Proc. Natl. Acad. Sci. U.S.A. 92: 6864-6867.

Bogler, D. J., J. C. Pires y J. Francisco-Ortega. 2006. Phylogeny of Agavaceae based on $\mathrm{ndhF}, \mathrm{rbcL}$, and ITS sequences: implications of molecular data for classification. Aliso 22: 313-328.

Clary, K. H. y B. B. Simpson. 1995. Systematics and character evolution of the genus Yucca L. (Agavaceae): evidence from morphology and molecular analyses. Bol. Soc. Bot. Méx. 56: 77-88.

Espejo, A. y A. R. López-Ferrari. 1993. Las monocotiledóneas mexicanas. Una sinopsis florística. Lista de referencia. Parte 1. Consejo Nacional de la Flora de México/ Universidad Autónoma Metropolitana. México, D.F. pp. 33-36.

García-Mendoza, A. y R. Galván. 1995. Riqueza de las familias Agavaceae y Nolinaceae en México. Bol. Soc. Bot. Méx. 56: 7-24.

Good-Avila, S. V., V. Souza, B. S. Gaut y L. E. Eguiarte. 2006. Timing and rate of speciation in Agave (Agavaceae). Proc. Natl. Acad. Sci. U.S.A. 103: 9124-9129.

Hernández-Sandoval, L. 1995. Análisis cladístico de la familia Agavaceae. Bol. Soc. Bot. Méx. 56: 57-68.

McVaugh, R. 1989. Flora Novo-Galiciana. Volume 15. Bromeliaceae to Dioscoreaceae. The University of Michigan Herbarium. Ann Arbor. 398 pp.

Piña-Luján, I. 1985. Consideraciones sobre el género Manfreda I. Cact. Suc. Mex. 30(2): 27-32.

Rocha, M., S. Good-Avila, F. Molina-Freaner, H. T. Arita, M. Castillo, A. García-Mendoza, A. Silva-Montellano, B. S. Gaut, V. Souza y L. E. Eguiarte. 2006. Pollination biology and adaptive radiation of Agavaceae, with special emphasis on the genus Agave. Aliso 22: 329-344.

Thiede, J. y U. Eggli. 1999. Einbeziehung von Manfreda Salisbury, Polianthes Linné und Prochnyanthes in Agave (Agavaceae). Kakteen Sukk. 50(5): 109-113.

Verhoek-Williams, S. 1978. Huaco and amole: a survey of the uses of Manfreda and Prochnyanthes. Econ. Bot. 32: 124-130.

Williams, S. 1998. Agavaceae. In: Kubitzki, K. (ed.). The families and genera of vascular plants III: flowering plants. Monocotyledons, Lilianae (except Orchidaceae). Springer. Berlin. pp. 60-70. 
